Wright State University

CORE Scholar

Mathematics and Statistics Faculty

Publications

Mathematics and Statistics

$9-2002$

\title{
Matroid Duality from Topological Duality in Surfaces of Nonnegative Euler Characteristic
}

\author{
Dan Slilaty \\ Wright State University - Main Campus, daniel.slilaty@wright.edu
}

Follow this and additional works at: https://corescholar.libraries.wright.edu/math

Part of the Applied Mathematics Commons, Applied Statistics Commons, and the Mathematics Commons

\section{Repository Citation}

Slilaty, D. (2002). Matroid Duality from Topological Duality in Surfaces of Nonnegative Euler Characteristic. Combinatorics Probability \& Computing, 11 (5), 515-528.

https://corescholar.libraries.wright.edu/math/23

This Article is brought to you for free and open access by the Mathematics and Statistics department at CORE Scholar. It has been accepted for inclusion in Mathematics and Statistics Faculty Publications by an authorized administrator of CORE Scholar. For more information, please contact library-corescholar@wright.edu. 


\title{
Matroid Duality from Topological Duality in Surfaces of Nonnegative Euler Characteristic
}

\section{DANIEL C. SLILATY}

Department of Mathematics and Statistics, Wright State University, Dayton, OH 45435, USA

(e-mail: dslilaty@math.wright.edu)

Received 13 June 2001; revised 11 February 2002

\begin{abstract}
Let $\mathrm{G}$ be a connected graph that is 2-cell embedded in a surface $\mathrm{S}$, and let $G^{*}$ be its topological dual graph. We will define and discuss several matroids whose element set is $E(G)$, for $\mathrm{S}$ homeomorphic to the plane, projective plane, or torus. We will also state and prove old and new results of the type that the dual matroid of $G$ is the matroid of the topological dual $G^{*}$.
\end{abstract}

\section{Introduction}

One of the most basic examples of matroid duality is the following. Let $G$ be a graph embedded in the plane and let $G^{*}$ be its topological dual graph. If $\mathscr{M}(G)$ is the cycle matroid of $G$, then the dual matroid $\mathscr{M}^{*}(G)=\mathscr{M}\left(G^{*}\right)$. If $G$ is a connected graph that is 2-cell embedded in a surface of demigenus $d>0$ (the demigenus is equal to 2 minus the Euler characteristic of the surface), then $\mathscr{M}^{*}(G) \neq \mathscr{M}\left(G^{*}\right)$ for the simple reason that

$$
\begin{aligned}
|V(G)|-|E(G)|+|F(G)| & =2-d, \\
(|V(G)|-1)+\left(\left|V\left(G^{*}\right)\right|-1\right) & =|E(G)|-d, \\
\operatorname{rk}(\mathscr{M}(G))+\operatorname{rk}\left(\mathscr{M}\left(G^{*}\right)\right) & =|E(G)|-d, \\
& \neq|E(G)|,
\end{aligned}
$$

while the rank of a matroid and its dual need to sum to the size of their element set. In this work we will use biased graphs and their matroids $[4,5]$ to describe methods of constructing matroids from $G$ embedded in the projective plane or torus of rank $|V(G)|$ that will give the desirable result that matroid duality comes from the topological dual of $G$. This duality will also yield a connection between closed cuts on surfaces, and matroid circuits and cocircuits. 


\section{Definitions and background}

\subsection{Graphs, embeddings and homology}

A graph is a pair $(V(G), E(G))$, in which $V(G)$ is a collection of topological points called vertices and $E(G)$ is a collection of topological 1-cells called edges. Each edge has two ends: an edge with both ends identified with a single vertex is called a loop and an edge with its ends identified with distinct vertices is called a link. If $S \subseteq E(G)$, then $G: S$ will denote the subgraph of $G$ consisting of the edges in $S$ and the vertices of $G$ incident to edges from $S$. Since, for any $H \subseteq G$ without vertices of degree zero, $H=G: E(H)$, we may, without being ambiguous, refer to a subgraph of $G$ without vertices of degree zero simply by referring to its edge set. We do this often.

Given an edge $e$, we denote an orientation of $e$ by $\vec{e}$ and the reverse orientation of $\vec{e}$ by $\vec{e}^{-1}$. A path is a graph that is a subdivision of a link. A circle is a graph that is a subdivision of a loop. We specify an orientation of a path $\gamma$ by $\vec{\gamma}$ and an orientation of a circle $C$ by $\vec{C}$. The reverse orientations are denoted by $\vec{\gamma}^{-1}$ and $\vec{C}^{-1}$, respectively. The edges of an oriented circle or path are considered to be oriented consistently with the oriented path or circle.

In this paper, all embeddings of graphs in surfaces will be 2-cell embeddings. That is, when $G$ is embedded in $\mathbf{S}, \mathbf{S} \backslash G$ is a disjoint union of open 2-cells. We use $F(G)$ to denote the set of open 2-cells (which we call faces) into which $G$ subdivides the surface $\mathrm{S}$ in which it is embedded. When $G$ is 2 -cell embedded in $\mathrm{S}$, it is necessary that $G$ is connected. The topological dual of $G$ is a graph embedded in S, denoted by $G^{*}$, which is constructed as follows. The vertex set $V\left(G^{*}\right)$ is in bijective correspondence with $F(G)$, where we denote the vertex corresponding to $f \in F(G)$ by $f^{*}$. The vertex $f^{*}$ is embedded in the interior of the face $f$. The edge set $E\left(G^{*}\right)$ is in bijective correspondence with $E(G)$, where the edge in $E\left(G^{*}\right)$ corresponding to $e \in E(G)$ is denoted by $e^{*}$. The edge $e^{*}$ has its ends connected to vertices $f_{1}^{*}$ and $f_{2}^{*}$ if and only if $f_{1}^{*} \neq f_{2}^{*}$, and the boundaries of faces $f_{1}$ and $f_{2}$ intersect on the edge $e$, or $f_{1}^{*}=f_{2}^{*}$ and $e$ is a boundary edge of the face $f_{1}$ twice. The edge $e^{*}$ is embedded connecting vertices (or vertex) $f_{1}^{*}$ and $f_{2}^{*}$ such that $e$ and $e^{*}$ intersect transversely at a point and $e^{*}$ does not intersect $G$ at any other point. From this definition, $G^{*}$ is connected when $G$ is connected, $G^{*}$ is 2-cell embedded in S and $\left(G^{*}\right)^{*}=G$. Given a set $X \subseteq E(G)$ we denote the corresponding subset of $E\left(G^{*}\right)$ as $X^{*}$. To avoid confusion, subsets of $E\left(G^{*}\right)$ will always be denoted with a superscript *. Figure 1 shows an embedding of $K_{4}$ in the torus with its topological dual constructed and embedded as defined above. The dual is drawn with the thicker edges.

Let $C(G)$ be the group of formal $\mathbb{Z}$-linear combinations of the oriented edges of $G$ modulo the relation $\vec{e}+\vec{e}^{-1}=0$. We call this the group of chains of $G$ over $\mathbb{Z}$. If $\vec{\omega}$ is an oriented walk in the graph $G$, then we also use the symbol $\vec{\omega}$ to denote the element of $C(G)$ consisting of the sum of the oriented edges of $\vec{\omega}$ all with coefficient $1 \in \mathbb{Z}$. Let $Z(G)$ be the subgroup of $C(G)$ generated by the oriented circles of $G$, and let $B(G)$ be the subgroup of $Z(G)$ generated by the oriented face boundaries of $G$. These, respectively, are called the group of cycles and the group of boundaries of $G$ over $\mathbb{Z}$. It is a well-known result of algebraic topology that the quotient group $H_{1}(\mathrm{~S})=Z(G) / B(G)$ is invariant, up to group isomorphism, for any graph $G$ that is 2-cell embedded in S. Given $G$ embedded 

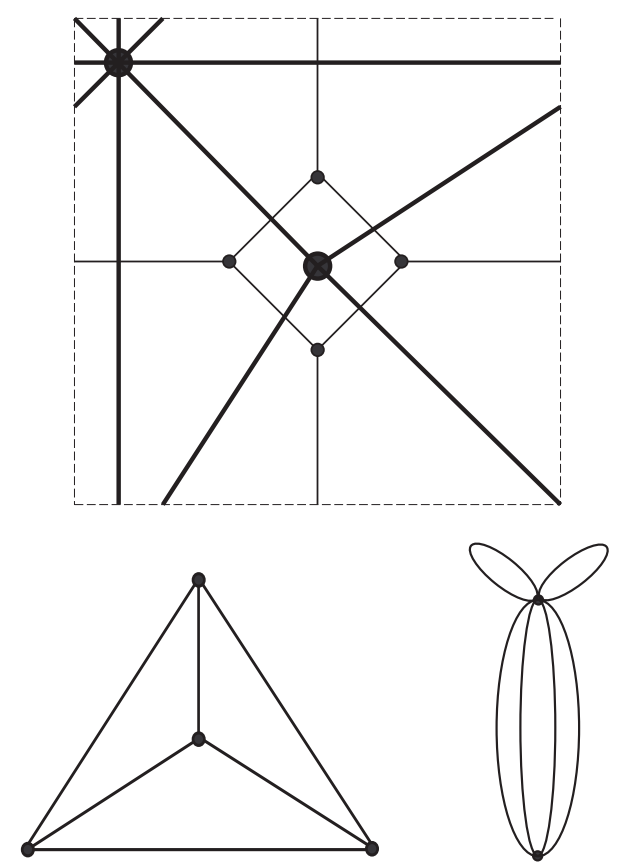

Figure 1 A depiction of $K_{4}$ (lower left) embedded in the torus (top) and drawn with the thinner lines, and its corresponding dual graph (lower right) drawn in the torus with thicker lines

in $\mathrm{S}$, let $i: Z(G) \rightarrow H_{1}(\mathrm{~S})$ be the natural homomorphism given by the embedding. Some immediate and important properties derived from the definition of the homomorphism $i: Z(G) \rightarrow H_{1}(\mathrm{~S})$ are given in Proposition 2.1 .

\section{Proposition 2.1.}

(1) If $\vec{C}$ is an oriented circle, then $i\left(\vec{C}^{-1}\right)=-i(\vec{C})$.

(2) For any theta graph with circles oriented as shown in Figure $2, i\left(\vec{C}_{1}\right)+i\left(\vec{C}_{2}\right)=i\left(\vec{C}_{1}+\right.$ $\left.\vec{C}_{2}\right)=i\left(\vec{C}_{3}\right)$.

It is well known that $H_{1}(\mathrm{~T}) \cong \mathbb{Z} \times \mathbb{Z}$ (here $\mathrm{T}$ denotes the torus). Proposition 2.2 can be found in [2, p. 214] and is used freely throughout this paper without further reference.

Proposition 2.2. There exists an oriented circle $C$ embedded in $\mathrm{T}$ with $i(\vec{C})=(m, n) \in H_{1}(\mathrm{~T})$ if and only if $(m, n)$ is a relatively prime pair of integers.

Let $\vec{C}$ and $\vec{D}$ be two oriented circles embedded in T such that $C \cap D$ is a finite collection of points. Each intersection point of $\vec{C}$ with $\vec{D}$ is one of three possible types: a clockwise transverse crossing, a counterclockwise transverse crossing, or a nontransverse crossing. Examples of clockwise and counterclockwise transverse crossings of $\vec{C}$ with $\vec{D}$ are shown in Figure 3. An example of a nontransverse crossing of two curves would be the intersection of the curves $y=0$ and $y=x^{2}$ in the $x y$-plane. The algebraic intersection $\vec{C} \cdot \vec{D}$ is 


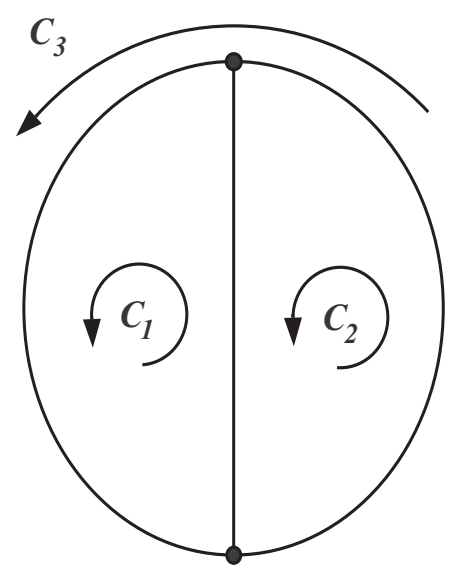

Figure 2 The desired orientations of the circles in the theta graph of Proposition 2.1
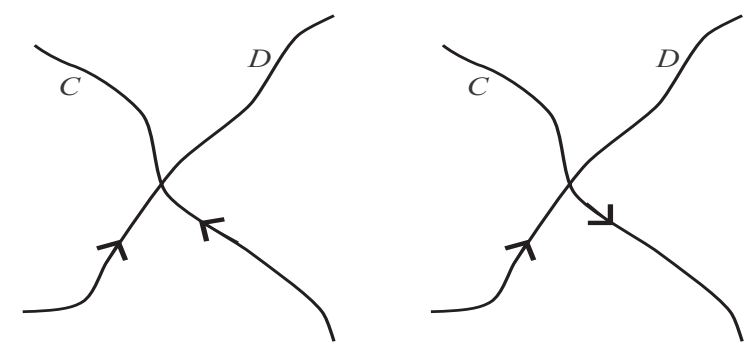

Figure 3 The left figure displays a clockwise transverse crossing of $\vec{C}$ with $\vec{D}$. The right is a counterclockwise transverse crossing of $\vec{C}$ with $\vec{D}$

the number of clockwise crossings of $\vec{C}$ with $\vec{D}$ minus the number of counterclockwise crossings of $\vec{C}$ with $\vec{D}$. Because of Propositions 2.3 and 2.4, the algebraic intersection of curves on a surface can be especially useful when studying graphs embedded in surfaces. Proposition 2.3 can be found in [2, Section 6.4.3].

Proposition 2.3. Let $\vec{C}$ and $\vec{D}$ be oriented circles embedded in $\mathrm{T}$ that intersect in a finite number of points. If $i(\vec{C})=\left(m_{1}, n_{1}\right)$ and $i(\vec{D})=\left(m_{2}, n_{2}\right)$, then

$$
\vec{C} \cdot \vec{D}= \pm\left|\begin{array}{ll}
m_{1} & n_{1} \\
m_{2} & n_{2}
\end{array}\right|
$$

Proposition 2.4. If $G$ is a graph embedded in a surface $S, C$ is the edge set of a circle in $G$, and $D^{*}$ is the edge set of a circle in $G^{*}$, then the following are true.

(1) $(G: C) \cap\left(G^{*}: D^{*}\right)=C \cap D^{*}$ is a finite collection of points, each point being a transverse crossing of a pair of dual edges $\left(e, e^{*}\right)$.

(2) $\left|C \cap D^{*}\right|=|C \cap D|$. 
In addition to the geometric meaning of the algebraic intersection of two oriented circles, Proposition 2.3 is even more useful, in that one may impose a multiplicative structure on $\mathbb{Z} \times \mathbb{Z} \cong H_{1}(\mathrm{~T})$ by defining the product $\left(m_{1}, n_{1}\right) \cdot\left(m_{2}, n_{2}\right)$ to be the $2 \times 2$ determinant in Proposition 2.3. Now the algebraic properties listed in Proposition 2.5 may be brought to bear on the elements of $Z(G)$ which include the oriented circles of $G$.

Proposition 2.5. For all $C, D, D^{\prime} \in Z(G)$, the following are true.

(1) $i(C) \cdot i(D)=-i(D) \cdot i(C)$.

(2) $i(C) \cdot i\left(D+D^{\prime}\right)=i(C) \cdot\left[i(D)+i\left(D^{\prime}\right)\right]=[i(C) \cdot i(D)]+\left[i(C) \cdot i\left(D^{\prime}\right)\right]$.

\subsection{Biased graphs and matroids derived from graphs embedded in the torus}

Biased graphs are discussed in [4]. We will state the relevant definitions. A collection of circles $L$ in a graph $G$ is called a linear class of circles if each theta subgraph of $G$ contains 0,1 , or 3 circles from $L$. A biased graph is a pair $(G, L)$ where $G$ is a graph and $L$ is a linear class of circles of $G$. Circles in $L$ are called balanced. A subgraph $H$ of $G$ is called balanced if all circles of $H$ are balanced; $H$ is called contrabalanced if all circles of $H$ are unbalanced.

A special case of a biased graph is an additively biased graph. This is a biased graph in which each theta subgraph contains either 1 or 3 balanced circles. A signed graph is a pair $(G, \sigma)$ in which $\sigma: E(G) \rightarrow\{+,-\}$. Given a signed graph $(G, \sigma)$, let $C_{+}$be the collection of circles in $G$ for which the product of signs on its edges is positive. In [3, Section 2] it is shown that the pair $\left(G, C_{+}\right)$is an additively biased graph; furthermore, any additively biased graph is of the form $\left(G, C_{+}\right)$for some signed graph $(G, \sigma)$.

We will define two biased graphs associated with $G$. First, let $H(G)$ be the collection of circles $C$ in $G$ such that $i(\vec{C})=-i\left(\vec{C}^{-1}\right)=\mathbf{0}$. The pair $\mathscr{H}(G)=(G, H(G))$ is a biased graph because Proposition 2.1(2) guarantees that a theta graph cannot have exactly two circles in the homology class $\mathbf{0} \in H_{1}(\mathrm{~T})$.

Second, let $A(G)$ be the collection of circles $C$ such that the vector dot product $i(\vec{C})$. $(1,1) \equiv 0 \bmod 2$ (which happens if and only if $i(\vec{C})=(m, n)$ and $m$ and $n$ have the same parity). The pair $\mathscr{A}(G)=(G, A(G))$ is an additively biased graph because Proposition 2.1(2) and the fact that $i\left(\vec{C}_{1}+\vec{C}_{2}\right) \cdot(1,1)=\left(i\left(\vec{C}_{1}\right)+i\left(\vec{C}_{2}\right)\right) \cdot(1,1)=i\left(\vec{C}_{1}\right) \cdot(1,1)+i\left(\vec{C}_{2}\right) \cdot(1,1)$ guarantee that a theta graph may only have 1 or 3 circles in $A(G)$.

Proposition 2.6. If $G$ is 2-cell embedded in $\mathrm{T}$, then $\mathscr{H}(G)$ and $\mathscr{A}(G)$ are both unbalanced biased graphs.

Proof. Since $H_{1}(\mathrm{~T})=Z(G) / B(G)$, the natural homomorphism $i: Z(G) \rightarrow H_{1}(\mathrm{~T}) \cong \mathbb{Z} \times Z$ is a surjection. So for each $(x, y) \in \mathbb{Z} \times Z$, there is $z \in Z(G)$ such that $i(z)=(a, b)$. By the definition of $Z(G)$, there are $\lambda_{1}, \ldots, \lambda_{t} \in \mathbb{Z}$ and oriented circles $\vec{C}_{1}, \ldots, \vec{C}_{t}$ in $G$ such that $z=\lambda_{1} \vec{C}_{1}+\cdots+\lambda_{t} \vec{C}_{t}$. So if we insist that $i(z) \neq \mathbf{0}$, or that $i(z) \cdot(1,1)=$ $\lambda_{1} i\left(\vec{C}_{1}\right) \cdot(1,1)+\cdots+\lambda_{t} i\left(\vec{C}_{t}\right) \cdot(1,1)$ is not even, then there must be some $i\left(\vec{C}_{j}\right) \neq \mathbf{0}$ or $i\left(\vec{C}_{j}\right) \cdot(1,1)$ that is not even. Thus there is an unbalanced circle in each of $\mathscr{H}(G)$ and $\mathscr{A}(G)$. 
We will consider three matroids associated with a biased graph $(G, L)$. Two of these matroids have element set $E(G)$ and the third has element set $E_{0}(G)=E(G) \cup e_{0}$. They are, respectively, the lift matroid $\mathscr{L}(G, L)$, the bias matroid $\mathscr{B}(G, L)$, and the complete lift matroid $\mathscr{L}_{0}(G, L)$.

These matroids are discussed in detail in [5]. We will review them in the next two paragraphs. The definitions of these matroids use the cyclomatic number of a graph $G$. It is the number of edges of $G$ outside a maximal forest: that is, $|E(G)|-|V(G)|+c(G)$, where $c(G)$ is the number of connected components of $G$. Also, in a matroid $M$, we will denote the rank of $X \subseteq E(M)$ by $\operatorname{rk}_{M}(X)$. The rank of a matroid $M$ is defined to be $\mathrm{rk}_{M}(E(M))$ but is denoted more simply by $\operatorname{rk}(M)$.

The circuits of the lift matroid $\mathscr{L}(G, L)$ are the edge sets of minimal balanced subgraphs of cyclomatic number 1 (i.e., balanced circles) and minimal contrabalanced subgraphs of cyclomatic number 2 (i.e., the union of two vertex-disjoint unbalanced circles, or the union of two unbalanced circles that intersect in a vertex or path and whose union is contrabalanced). See Figure 4. Proposition 2.7 is from [5, Theorem 3.1(j)].

Proposition 2.7. Given $X \subseteq E(G)$,

$$
\operatorname{rk}_{\mathscr{L}(G, L)}(X)=\left\{\begin{array}{ll}
|V(G: X)|-c(G: X) & \text { if } G: X \text { is unbalanced } \\
|V(G: X)|-c(G: X)-1 & \text { if } G: X \text { is balanced }
\end{array}\right\} .
$$

The complete lift matroid $\mathscr{L}_{0}(G, L)$ is defined to be the lift matroid of the biased graph $(G, L)_{0}$, which is $(G, L)$ together with a new vertex $v_{0}$ attached to the unbalanced loop $e_{0}$. Proposition 2.8 is apparent from the definitions of $\mathscr{L}(G, L)$ and $\mathscr{L}_{0}(G, L)$.

\section{Proposition 2.8.}

(1) $\mathscr{L}_{0}(G, L) \backslash e_{0}=\mathscr{L}(G, L)$.

(2) $\mathscr{L}_{0}(G, L) / e_{0}=\mathscr{M}(G)$.

The circuits of the bias matroid $\mathscr{B}(G, L)$ are the edge sets of minimal balanced subgraphs of cyclomatic number 1 (i.e., balanced circles) and minimal connected contrabalanced subgraphs of cyclomatic number 2 (i.e., the union of two vertex-disjoint unbalanced circles and a connecting path between them or the union of two unbalanced circles that intersect in a vertex or path and whose union is contrabalanced). See Figure 5. Proposition 2.9 is from [5, Theorem 2.1(j)].

Proposition 2.9. Given $X \subseteq E(G)$,

$$
\operatorname{rk}_{\mathscr{B}(G, L)}(X)=|V(G: X)|-b(G: X),
$$

where $b(G: X)$ is the number of connected components of $G: X$ that are balanced.

Proposition 2.10 is evident from the definitions of $\mathscr{B}(G, L)$ and $\mathscr{L}(G, L)$.

Proposition 2.10. If $(G, L)$ is a biased graph, then $\mathscr{B}(G, L)=\mathscr{L}(G, L)$ if and only if there do not exist 2 vertex-disjoint unbalanced circles in $(G, L)$. 

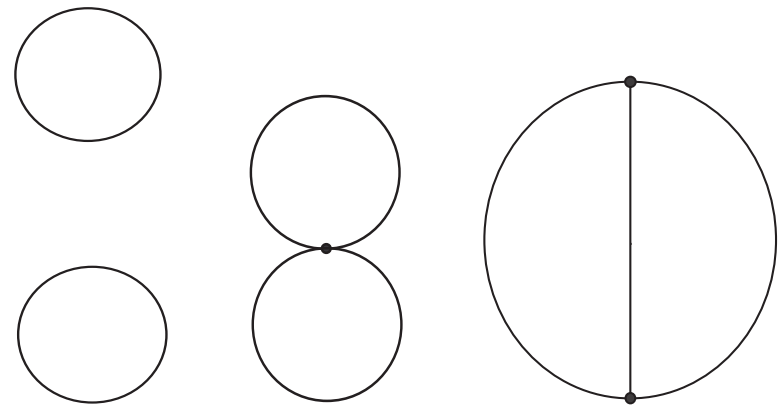

Figure 4 Lift matroid circuits are edge sets of balanced circles and edge sets of contrabalanced subgraphs that are subdivisions of one of the above graphs
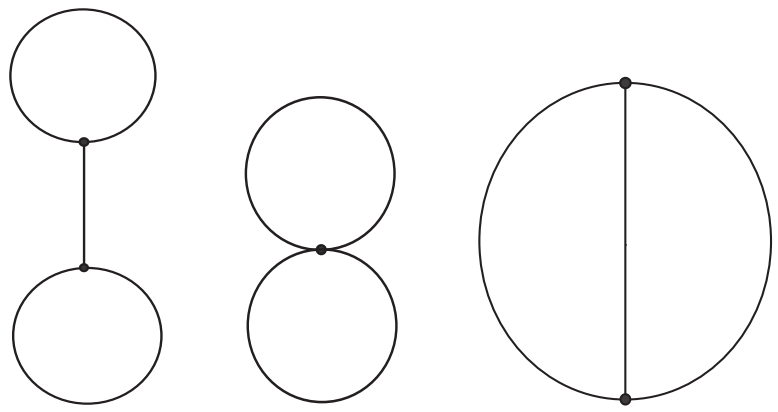

Figure 5 Bias matroid circuits are edge sets of balanced circles and edge sets of contrabalanced subgraphs that are subdivisions of one of the above graphs

\subsection{Matroid duality}

Let $M$ be a matroid on the element set $E$. If $\mathfrak{B}$ is the set of bases of $M$, then the set of cobases of $M$ is $E \backslash \mathfrak{B}=\{E \backslash B: B \in \mathfrak{B}\}$. The dual matroid $M^{*}$ is normally defined to be the matroid on $E$ whose set of bases is $E \backslash \mathfrak{B}$ and whose set of cobases is $\mathfrak{B}$. For our purposes, it will be more convenient to do the following. Let $E^{*}$ be a set in bijective correspondence with $E$ by the map $e \mapsto e^{*}$. The subset of $E^{*}$ corresponding to $X \subseteq E$ will be denoted by $X^{*}$; additionally, subsets of $E^{*}$ will always be written with a * superscript. Define $M^{*}$ to be the matroid on $E^{*}$ whose set of bases is $(E \backslash \mathfrak{B})^{*}$ and whose set of cobases is $\mathfrak{B}^{*}$. Proposition 2.11 is a characterization of matroid duality.

Proposition 2.11. If $M$ is a matroid on $E$ and $N$ is a matroid on $E^{*}$, then $M^{*}=N$ if and only if $\operatorname{rk}(M)+\operatorname{rk}(N)=|E|$ and, for each circuit $C$ of $M$ and each circuit $D^{*}$ of $N$, $|C \cap D| \neq 1$.

Proof. The conditions on rank and circuit intersections are well known necessary conditions for $M^{*}=N$. Conversely, let $B$ be a basis of $M$. Since $\operatorname{rk}(M)+\operatorname{rk}(N)=|E|$, showing that $(E \backslash B)^{*}$ is independent in $N$ will imply that $(E \backslash B)^{*}$ is a basis of $N$. This will prove that $M^{*}=N$.

By way of contradiction, if we assume that $(E \backslash B)^{*}$ is a dependent set in $N$, there exists $D^{*} \subseteq(E \backslash B)^{*}$ that is a circuit of $N$. Pick $e \in D$. Since $B$ is a basis of $M$ and $e \notin B$, there 
exists $C \subseteq B \cup\{e\}$ that is a circuit of $M$ and contains $e$. This gives us the contradiction $|C \cap D|=1$.

\subsection{Matroid duality from graphs embedded in the plane and projective plane}

Proposition 2.11 along with Proposition 2.4 are excellent tools for proving results about matroid duality from graphs embedded in surfaces. Using them will prove two known results (Theorem 2.12 and Corollary 2.16) and one closely related result (Theorem 2.15).

Theorem 2.12. If $G$ be a connected graph embedded in the plane, then $\mathscr{M}^{*}(G)=\mathscr{M}\left(G^{*}\right)$.

Proof. Since $G$ is connected and embedded in the plane, $|V(G)|-|E(G)|+|F(G)|=2$. By definition of $G^{*},|V(G)|-|E(G)|+\left|V\left(G^{*}\right)\right|=2$. Thus

$$
\begin{aligned}
& (|V(G)|-1)+\left(\left|V\left(G^{*}\right)\right|-1\right)=|E(G)|, \\
& \operatorname{rk}(\mathscr{M}(G))+\operatorname{rk}\left(\mathscr{M}\left(G^{*}\right)\right)=|E(G)|,
\end{aligned}
$$

which is the first condition from Proposition 2.11 needed to prove that $\mathscr{M}^{*}(G)=\mathscr{M}\left(G^{*}\right)$. The second and last condition of Proposition 2.11 is that, for each circle $C$ in $G$ and circle $D^{*}$ in $G^{*},|E(C) \cap E(D)| \neq 1$. By Proposition $2.4,|E(C) \cap E(D)| \neq 1$ if and only if $\left|C \cap D^{*}\right| \neq 1$. Now the Jordan curve theorem states that every simple closed curve in the plane separates the plane into two regions. Since every intersection of $C$ and $D^{*}$ is transverse, $\left|C \cap D^{*}\right|$ is even. Thus $|C \cap D| \neq 1$, as required.

Corollary 2.13. If $G$ is a graph embedded in the plane and $X \subseteq E(G)$, then $G: X$ separates the plane if and only if $X^{*}$ separates $G^{*}$.

Proof. It is known that the cocircuits of $\mathscr{M}(G)$ are the bonds of $G$. Since $\mathscr{M}^{*}(G)=\mathscr{M}\left(G^{*}\right)$, $C$ is a circuit of $\mathscr{M}(G)$ iff $C^{*}$ is a cocircuit of $\mathscr{M}\left(G^{*}\right)$. Thus $G: X$ separates the plane iff $G: X$ contains a circle iff $X^{*}$ contains a bond of $G^{*}$ iff $X^{*}$ separates $G^{*}$.

Corollary 2.16 below is an unpublished result of Thomas Zaslavsky from [6]. It follows from Theorem 2.15, Proposition 2.8, and the fact that, for any matroid $M$ and $e \in E(M)$, $(M / e)^{*}=M^{*} \backslash e^{*}$. Before presenting these results, we note a few facts about graphs embedded in the projective plane, which we denote by $\mathrm{P}_{2}$.

Proposition 2.14. If $G$ is a connected graph that is 2-cell embedded in the projective plane, then

(1) $H_{1}\left(\mathrm{P}_{2}\right)=Z(G) / B(G) \cong \mathbb{Z}_{2}$,

(2) $C$ is a circle in $G$ that separates $\mathrm{P}_{2}$ if and only if $i(C)=0$, and

(3) if $C$ and $C^{\prime}$ are circles such that $i(C)=i\left(C^{\prime}\right)=1$, then $C \cap C^{\prime} \neq \emptyset$.

Now let $H(G)$ be the collection of circles in $G$ such that $i(C)=0$ (i.e., $H(G)$ is the collection of circles in $G$ that separate $\mathrm{P}_{2}$ ). It follows from Propositions 2.1, 2.14, and 2.10 that $\mathscr{H}(G)=(G, H(G))$ is an additively biased graph for which $\mathscr{B}(\mathscr{H}(G))=\mathscr{L}(\mathscr{H}(G))$. 
Theorem 2.15. If $G$ is a graph that is 2-cell embedded in the projective plane, then $\mathscr{L}_{0}^{*}(\mathscr{H}(G))=\mathscr{L}_{0}\left(\mathscr{H}\left(G^{*}\right)\right)$.

Proof. Since $G^{*}$ is 2-cell embedded in the projective plane, $G$ is connected and $i: Z(G) \rightarrow$ $H_{1}\left(\mathrm{P}_{2}\right)=Z(G) / B(G)$ is onto. Thus there must be an unbalanced circle in $\mathscr{H}(G)$. Thus $\operatorname{rk}\left(\mathscr{L}_{0}(\mathscr{H}(G))\right)=|V(G)|$. Similarly, $\operatorname{rk}\left(\mathscr{L}_{0}\left(\mathscr{H}\left(G^{*}\right)\right)\right)=\left|V\left(G^{*}\right)\right|$. Since $G$ is connected and is 2-cell embedded in the projective plane, we have $|V(G)|-|E(G)|+|F(G)|=1$. Thus

$$
\begin{array}{ll}
|V(G)|+\left|V\left(G^{*}\right)\right| & =|E(G)|+1 \\
\operatorname{rk}\left(\mathscr{L}_{0}(\mathscr{H}(G))\right)+\operatorname{rk}\left(\mathscr{L}_{0}\left(\mathscr{H}\left(G^{*}\right)\right)\right) & =\left|E_{0}(G)\right| .
\end{array}
$$

Our result will now follow by showing that, for any circuit $C$ in $\mathscr{L}_{0}(\mathscr{H}(G))$ and any circuit $D^{*}$ in $\mathscr{L}_{0}\left(\mathscr{H}\left(G^{*}\right)\right)$, we have $\left|C \cap D^{*}\right| \neq 1$. Now, without loss of generality, either $C$ is a balanced circle or neither $C$ nor $D$ is a balanced circle.

Case 1: Recall that $C$ is a balanced circle because $i(C)=0$. Thus $C$ separates $\mathrm{P}_{2}$. Since $D$ is either a circle or the union of two edge-disjoint circles and since $G$ and $G^{*}$ only intersect in transversely crossing dual edge pairs, $\left|C \cap D^{*}\right|$ is even. In particular $\left|C \cap D^{*}\right| \neq 1$.

Case 2: Since both $C$ and $D^{*}$ are not balanced circles, $C$ is the union of two edge-disjoint circles in $G$ and $D^{*}$ is the union of two edge-disjoint circles in $G^{*}$. Since $G$ and $G^{*}$ only intersect in transversely crossing dual edge pairs, we get the following: $\left|C \cap D^{*}\right| \geqslant 4$ when $e_{0} \notin C$ and $e_{0}^{*} \notin D^{*},\left|C \cap D^{*}\right| \geqslant 2$ when $e_{0} \notin C$ if and only if $e_{0}^{*} \in D^{*}$, and $\left|C \cap D^{*}\right| \geqslant 2$ when $e_{0} \in C$ and $e_{0}^{*} \in D^{*}$. In all cases $\left|C \cap D^{*}\right| \neq 1$.

Corollary 2.16. If $G$ is a graph that is 2-cell embedded in the projective plane, then $\mathscr{M}^{*}(G)=$ $\mathscr{L}\left(\mathscr{H}\left(G^{*}\right)\right)$.

\section{Lift matroid duality from topological duality}

Theorems 3.1 and 3.2 are both similar to the well-known result that $\mathscr{M}^{*}(G)=\mathscr{M}\left(G^{*}\right)$ for a graph $G$ embedded in the plane.

Theorem 3.1. If $G$ is a connected graph that is 2-cell embedded in the torus, then $\mathscr{L}^{*}(\mathscr{H}(G))=\mathscr{L}\left(\mathscr{H}\left(G^{*}\right)\right)$.

Theorem 3.2. If $G$ is a connected graph that is 2-cell embedded in the torus, then $\mathscr{L}^{*}(\mathscr{A}(G))=\mathscr{L}\left(\mathscr{A}\left(G^{*}\right)\right)$.

Before the proofs of Theorems 3.1-3.2 we present Lemmas 3.3-3.5.

Lemma 3.3. If $G$ is a connected graph that is 2-cell embedded in the torus, then

(1) the matroids $\mathscr{B}(\mathscr{H}(G)), \mathscr{L}(\mathscr{H}(G)), \mathscr{B}(\mathscr{A}(G))$, and $\mathscr{L}(\mathscr{A}(G))$ all have rank $|V(G)|$, and

(2) $|V(G)|-|E(G)|+|F(G)|=0$. 
Proof. Part (1) follows from Proposition 2.6 and the definition of rank in the bias and lift matroids. Part (2) is Euler's formula.

Lemma 3.4. Let $G$ be a graph that is 2-cell embedded in the torus.

(1) If $C$ and $D$ are vertex-disjoint circles in $G$ with each $i(\vec{C}), i(\vec{D}) \neq \mathbf{0}$, then $i(\vec{C})= \pm i(\vec{D})$.

(2) If $\Theta$ is a contrabalanced theta graph in $\mathscr{H}(G)$ with circles $C_{1}, C_{2}, C_{3}$, then $i\left(\overrightarrow{\vec{C}}_{j}\right) \neq$ $\pm i\left(\vec{C}_{k}\right)$ for each $j \neq k$.

Proof. (1) Since $C$ and $D$ are vertex-disjoint, $\vec{C} \cdot \vec{D}=0$. A $2 \times 2$ determinant with nonzero rows is zero is if and only if the first row is a constant multiple of the second. If this multiple is anything other than \pm 1 , then either $i(\vec{C})$ or $i(\vec{D})$ is not a relatively prime pair.

(2) By way of contradiction, say that $i\left(\vec{C}_{1}\right)=i\left(\vec{C}_{2}\right)$. Thus $i\left(\vec{C}_{3}\right)= \pm i\left(\vec{C}_{1}\right) \pm i\left(\vec{C}_{2}\right)=0$ or $\pm 2 i\left(\vec{C}_{1}\right)$; however, $i\left(\vec{C}_{3}\right) \neq \mathbf{0}$ because $\Theta$ is contrabalanced, and $i\left(\vec{C}_{3}\right) \neq \pm 2 i\left(\vec{C}_{1}\right)$ because $i\left(\vec{C}_{3}\right)$ is a relatively prime pair of integers.

Lemma 3.5. If $G$ is a graph embedded in $\mathrm{S}, C$ is a circle in $G$, and $D^{*}$ is a circle in $G^{*}$, then

(1) $\left|C \cap D^{*}\right|=1$ implies that $\vec{C} \cdot \vec{D}= \pm 1$, and

(2) $\left|C \cap D^{*}\right|$ is even if and only if $\vec{C} \cdot \vec{D}$ is even.

Proof. Both parts follow from the fact that $G$ and $G^{*}$ only intersect in transversely crossing edge pairs $e, e^{*}$.

Proof of Theorem 3.1. To prove our theorem we will use Proposition 2.11. First, Euler's formula states that $|V(G)|-|E(G)|+|F(G)|=0$ when $G$ is a 2-cell embedding. Since $G$ and $G^{*}$ are unbalanced (by Proposition 2.6), $\operatorname{rk}(\mathscr{L}(\mathscr{H}(G)))=|V(G)|$ and $\operatorname{rk}\left(\mathscr{L}\left(\mathscr{H}\left(G^{*}\right)\right)\right)=$ $|F(G)|$. Thus $\operatorname{rk}(\mathscr{L}(\mathscr{H}(G)))+\operatorname{rk}\left(\mathscr{L}\left(\mathscr{H}\left(G^{*}\right)\right)\right)=|E(G)|$. So, to complete the proof we need only show that $\left|C \cap D^{*}\right| \neq 1$, for any lift circuit $C$ in $\mathscr{H}(G)$ and lift circuit $D^{*}$ in $\mathscr{H}\left(G^{*}\right)$. We break the proof into two cases. In the first case, $C$ is a balanced circle and, in the second case, both $C$ and $D$ are not balanced circles.

Case 1: Since $C$ is a balanced circle in $\mathscr{H}, i(\vec{C})=\mathbf{0}$. Thus, for any circle $D_{0}^{*}$ in $D^{*}, \vec{C} \cdot \vec{D}_{0}^{*}$ is even. Thus $\left|E(C) \cap E\left(D_{0}\right)\right| \neq 1$.

Case 2: Since $C$ and $D^{*}$ are not balanced circles we may write $C=C_{1} \cup C_{2}$ and $D^{*}=D_{1}^{*} \cup D_{2}^{*}$ where $\left(C_{1}, C_{2}\right)$ and $\left(D_{1}^{*}, D_{2}^{*}\right)$ are modular pairs of circles in nonzero homology classes. Without loss of generality, we may split the remainder of the proof into the following four subcases:

$$
\begin{aligned}
& i\left(\vec{C}_{1}\right)= \pm i\left(\vec{C}_{2}\right)= \pm i\left(\vec{D}_{1}\right)= \pm i\left(\vec{D}_{2}\right), \\
& i\left(\vec{C}_{1}\right)= \pm i\left(\vec{C}_{2}\right) \neq \pm i\left(\vec{D}_{1}\right)= \pm i\left(\vec{D}_{2}\right), \\
& i\left(\vec{C}_{1}\right)= \pm i\left(\vec{C}_{2}\right) \text { and } \pm i\left(\vec{D}_{1}\right) \neq \pm i\left(\vec{D}_{2}\right), \\
& i\left(\vec{C}_{1}\right) \neq \pm i\left(\vec{C}_{2}\right) \text { and } \pm i\left(\vec{D}_{1}\right) \neq \pm i\left(\vec{D}_{2}\right) .
\end{aligned}
$$


Case 2.1: By Lemma 3.4, $E\left(C_{1}\right) \cap E\left(C_{2}\right)=E\left(D_{1}^{*}\right) \cap E\left(D_{2}^{*}\right)=\emptyset$. Since each $\vec{C}_{j} \cdot \vec{D}_{k}^{*}=0$, each $\left|E\left(C_{j}\right) \cap E\left(D_{k}\right)\right|$ is even. Thus $|E(C) \cap E(D)|=\sum_{j, k \in\{1,2\}}\left|E\left(C_{j}\right) \cap E\left(D_{k}\right)\right|$ is even (in particular, $|E(C) \cap E(D)| \neq 1)$.

Case 2.2: By Lemma 3.4, $E\left(C_{1}\right) \cap E\left(C_{2}\right)=E\left(D_{1}^{*}\right) \cap E\left(D_{2}^{*}\right)=\emptyset$. Since each $\vec{C}_{j} \cdot \vec{D}_{k}^{*} \neq 0$, each $\left|E\left(C_{j}\right) \cap E\left(D_{k}\right)\right| \geqslant 1$. Thus $|E(C) \cap E(D)|=\sum_{j, k \in\{1,2\}}\left|E\left(C_{j}\right) \cap E\left(D_{k}\right)\right| \geqslant 4$.

Case 2.3: By Lemma 3.4, $E\left(C_{1}\right) \cap E\left(C_{2}\right)=\emptyset$. Without loss of generality, $i\left(\vec{C}_{1}\right) \neq \pm i\left(\vec{D}_{1}^{*}\right)$. Thus each $\vec{C}_{j} \cdot \vec{D}_{1}^{*} \neq 0$, and $|E(C) \cap E(D)| \geqslant \sum_{j \in\{1,2\}}\left|E\left(C_{j}\right) \cap E\left(D_{1}\right)\right| \geqslant 2$.

Case 2.4: Without loss of generality $\vec{C}_{1} \cdot \vec{D}_{1}^{*} \neq 0$ and $\vec{C}_{2} \cdot \vec{D}_{2}^{*} \neq 0$. We may now conclude that $|E(C) \cap E(D)| \geqslant 2$ unless we have $E\left(C_{1}\right) \cap E\left(D_{1}\right)=E\left(C_{2}\right) \cap E\left(D_{2}\right)=\{e\}$. In the latter case, $C$ must be a contrabalanced theta graph in $\mathscr{H}$, because $E\left(C_{1}\right) \cap E\left(C_{2}\right) \neq \emptyset$. Consider $C_{1}+C_{2}$, which is the third circle in $C$. We cannot have $\left(\vec{C}_{1}+\vec{C}_{2}\right) \cdot \vec{D}_{1}^{*}=$ $\left(\vec{C}_{1}+\vec{C}_{2}\right) \cdot \vec{D}_{2}^{*}=0$, or else $i\left(\vec{D}_{1}^{*}\right)= \pm i\left(\vec{C}_{1}+\vec{C}_{2}\right)= \pm i\left(\vec{D}_{2}^{*}\right)$ while we assumed $i\left(\vec{D}_{1}^{*}\right) \neq \pm i\left(\vec{D}_{2}^{*}\right)$. Thus $\left|E\left(C_{1}+C_{2}\right) \cap E\left(D_{1}\right)\right| \geqslant 1$ or $\left|E\left(C_{1}+C_{2}\right) \cap E\left(D_{2}\right)\right| \geqslant 1$ and, since $e \in E\left(C_{1}\right) \cap E\left(C_{2}\right)$, we may now conclude that $|E(C) \cap E(D)| \geqslant 2$.

Proof of Theorem 3.2. First, $\operatorname{rk}(\mathscr{L}(\mathscr{A}(G)))+\operatorname{rk}\left(\mathscr{L}\left(\mathscr{A}\left(G^{*}\right)\right)\right)=|E(G)|$ follows from the same argument as in the first paragraph of the proof of Theorem 3.1. So let $C$ be a lift circuit of $\mathscr{A}(G)$ and $D^{*}$ be a lift circuit of $\mathscr{A}\left(G^{*}\right)$. We will show that $\left|E(C)^{*} \cap E(D)\right| \neq 1$. Recall that an additively biased graph contains no contrabalanced theta graphs. Thus a lift circuit is either a balanced circle or the union of two edge-disjoint unbalanced circles. We divide the remainder of the proof into three cases: $C$ and $D^{*}$ are both balanced circles, $C$ is a balanced circle and $D^{*}$ is not, $C$ and $D^{*}$ are not balanced circles.

Case 1: Here $i(\vec{C}) \cdot(1,1)$ and $i\left(\vec{D}^{*}\right) \cdot(1,1)$ are both even. Thus $i(\vec{C})$ and $i\left(\vec{D}^{*}\right)$ are both pairs of odd integers, which implies the $2 \times 2$ determinant $\vec{C} \cdot \vec{D}^{*}$ is even. Thus $|E(C) \cap E(D)|$ is even, and $|E(C) \cap E(D)| \neq 1$.

Case 2: Here $i(\vec{C})$ is a pair of odd integers and $D^{*}$ is the union of two edge-disjoint circles $D_{1}^{*}$ and $D_{2}^{*}$ such that each $i\left(\vec{D}_{j}^{*}\right)$ is a pair of integers of different parity. Thus the $2 \times 2$ determinant $\vec{C} \cdot \vec{D}_{j}^{*}$ is odd. Thus each $\left|E(C) \cap E\left(D_{j}\right)\right| \geqslant 1$, whence $|E(C) \cap E(D)| \geqslant 2$.

Case 3: Here $C$ is the union of edge-disjoint circles $C_{1}$ and $C_{2}$ and $D^{*}$ is the union of two edge-disjoint circles $D_{1}^{*}$ and $D_{2}^{*}$. The details of the remainder of the proof are contained in the proof of Theorem 3.1, Case 2.

\subsection{The topological action of lift circuits on the torus}

Theorem 3.1 tells us that if $C$ is a lift circuit of $\mathscr{H}(G)$, then $C^{*}$ is a lift cocircuit of $\mathscr{H}\left(G^{*}\right)$. In a matroid $M, H \subseteq E$ is a hyperplane if and only if $E \backslash H$ is a cocircuit. Thus a lift cocircuit in a biased graph is a minimal edge set $D$ such that $\operatorname{rk}_{\mathscr{L}(G, L)}(E(G) \backslash D)=\operatorname{rk}_{\mathscr{L}(G, L)}(E(G))-1$. Thus $D$ is the minimal edge set that either disconnects $G$ or whose removal leaves a balanced subgraph of $(G, L)$. Given this and Proposition 2.7, we have Proposition 3.6. 
Proposition 3.6. If $C$ is a lift cocircuit of $(G, L)$, then

(1) $G \backslash C$ is a connected and balanced subgraph of $(G, L)$, or

(2) $C$ is a bond of $G$ separating connected subgraphs $G_{1}$ and $G_{2}$, no more than one of which is balanced.

Combining Theorem 3.1 and Proposition 3.6 yields Theorem 3.7.

Theorem 3.7. If $G$ is 2-cell embedded in the torus $\mathrm{T}$ and $C$ is a lift circuit of $\mathscr{H}(G)$, then either $\mathrm{T} \backslash C$ has two connected components, no more than one of which is homeomorphic to a disk, or $\mathbf{T} \backslash C$ is homeomorphic to a disk; furthermore, $\mathrm{T} \backslash C$

(1) has two connected components if and only if $C$ is a balanced circle or $C$ is the union of two unbalanced circles $C_{1}$ and $C_{2}$ with $i\left(\vec{C}_{1}\right)= \pm i\left(\vec{C}_{2}\right)$, and

(2) is homeomorphic to a disk if and only if $C$ is the union of two unbalanced circles $C_{1}$ and $C_{2}$ with $\vec{C}_{1} \cdot \vec{C}_{2}= \pm 1$.

Proof. The collection of 2-cells $F(G)$ is a collection of open polygons whose boundary edges are identified in distinct pairs with the edges of $G$ which then yields a torus. Each $e^{*} \in E\left(G^{*}\right)$ represents an identification of one distinct pair of boundary edges of faces $f_{1}$ and $f_{2}$ (which are not necessarily distinct) with the edge $e \in E(G)$. The action of removing $e^{*}$ from $G^{*}$ is thus equivalent to removing the identification of the boundary edges of $f_{1}$ and $f_{2}$ with $e \in E(G)$ (i.e., removing $e^{*}$ from $G^{*}$ is equivalent to cutting the torus along $e$ from $G)$. Also, if $X^{*} \subseteq E\left(G^{*}\right)$, then each connected component $K^{*}: G^{*}$ of $G^{*} \backslash X^{*}$ will correspond to a connected component, $\mathrm{T}_{K}$, say, obtained by cutting the torus along $X \subseteq E(G)$; furthermore $\mathrm{T}_{K}$ is constructed from the faces of $F(G)$ corresponding to the vertices of $K^{*}: G^{*}$, with the boundary identifications represented by the edges of $K^{*}$. Since a 2-cell is the only surface with boundary (up to homeomorphism) that has trivial first homology group, $\mathrm{T}_{K}$ will be a 2-cell if and only if $K^{*}$ is a balanced subgraph of $\mathscr{H}\left(G^{*}\right)$. This, together with Theorem 3.1 and Proposition 3.6, implies our desired results.

\section{Bias matroid duality from topological duality}

Theorem 4.1. If $G$ is 2-cell embedded in the torus, then $\mathscr{B}^{*}(\mathscr{H}(G))=\mathscr{B}\left(\mathscr{H}\left(G^{*}\right)\right)$.

Proof. First, $\operatorname{rk}(\mathscr{B}(\mathscr{H}(G)))+\operatorname{rk}\left(\mathscr{B}\left(\mathscr{H}\left(G^{*}\right)\right)\right)=|E(G)|$ follows from the same argument as in the first paragraph of the proof of Theorem 3.1.

Second, let $C$ be a circuit of $\mathscr{B}(\mathscr{H}(G))$ and let $D^{*}$ be a circuit of $\mathscr{B}\left(\mathscr{H}\left(G^{*}\right)\right)$. A circuit $C^{\prime}$ in a bias matroid $\mathscr{B}(G, L)$ is also a circuit in the lift matroid $\mathscr{L}(G, L)$, except when $C^{\prime}$ consists of two vertex-disjoint unbalanced circles in a connecting path (called a loose handcuff). In this case $C^{\prime}$ is the union of a circuit in $\mathscr{L}(G, L)$ along with a connecting path. Thus we may use the exact same arguments as in the proof of Theorem 3.1 to show that $|E(C) \cap E(D)| \neq 1$, except when either $C$ or $D^{*}$ is a loose handcuff. In the case where either $C$ or $D^{*}$ is a loose handcuff let $C_{\mathscr{L}}$ and $D_{\mathscr{L}}^{*}$ be the unique lift circuits contained in $C$ and $D$, respectively. By Theorem 3.1, $\left|E\left(C_{\mathscr{L}}\right) \cap E\left(D_{\mathscr{L}}\right)\right| \neq 1$. So we may now conclude 
that $|E(C) \cap E(D)| \neq 1$ unless $\left|E\left(C_{\mathscr{L}}\right) \cap E\left(D_{\mathscr{L}}\right)\right|=0$. Without loss of generality we split the remainder of the proof into the following three cases where, in each one, $C$ is a loose handcuff and $\left|E\left(C_{\mathscr{L}}\right) \cap E\left(D_{\mathscr{L}}\right)\right|=0$ : in Case $1, D^{*}$ is a balanced circle; in Case 2, $D^{*}$ contains two circles $D_{1}^{*}$ and $D_{2}^{*}$ for which $i\left(\vec{D}_{1}^{*}\right) \neq \pm i\left(\vec{D}_{2}^{*}\right)$; in Case $3, D^{*}$ contains two circles $D_{1}^{*}$ and $D_{2}^{*}$ for which $i\left(\vec{D}_{1}^{*}\right)= \pm i\left(\vec{D}_{2}^{*}\right)$. In each of the three cases, write $C=C_{1} \cup C_{2} \cup \gamma$ in which $C_{\mathscr{L}}=C_{1} \cup C_{2}$ and $\gamma$ is the connecting path of the loose handcuff. By Lemma 3.4(1), $i\left(\vec{C}_{1}\right)= \pm i\left(\vec{C}_{2}\right)$.

Case 1: Since $D$ is a balanced circle, $D$ bounds a disk in the torus. Since $\mid E\left(C_{\mathscr{L}}\right) \cap$ $E\left(D_{\mathscr{L}}\right) \mid=0$, both endpoints of the path $\gamma$ are not in the interior of the disk bounded by $D$. Thus $|E(\gamma) \cap E(D)|$ is even, whence $|E(C) \cap E(D)|$ is even. In particular, $\mid E(C) \cap$ $E(D) \mid \neq 1$.

Case 2: Since $i\left(\vec{D}_{1}^{*}\right) \neq \pm i\left(\vec{D}_{2}^{*}\right)$, there exist $j$ and $k$ such that $\vec{C}_{j} \cdot \vec{D}_{k}^{*}$ is odd, which cannot happen when $\left|E\left(C_{\mathscr{L}}\right) \cap E\left(D_{\mathscr{L}}\right)\right|=0$. Thus this case does not occur when $\mid E\left(C_{\mathscr{L}}\right) \cap$ $E\left(D_{\mathscr{L}}\right) \mid=0$.

Case 3: Since $i\left(\vec{D}_{1}^{*}\right)= \pm i\left(\vec{D}_{2}^{*}\right)$ and $\left|E\left(C_{\mathscr{L}}\right) \cap E\left(D_{\mathscr{L}}\right)\right|=0$, we deduce that each $\vec{D}_{j}^{*} \cdot \vec{C}_{1}=0$. Thus $i\left(\vec{D}_{1}^{*}\right)= \pm i\left(\vec{D}_{2}^{*}\right)= \pm i\left(\vec{C}_{1}\right)= \pm i\left(\vec{C}_{2}\right)$. By Theorem 3.7, $C_{\mathscr{L}}$ separates the torus into two connected components, call them $T_{1}$ and $T_{2}$, both of whose boundaries are the two vertex-disjoint circles $C_{1}$ and $C_{2}$. Without loss of generality, the connecting path $\gamma$ is contained in $\mathrm{T}_{1}$ with its endpoints connecting the boundary components $C_{1}$ and $C_{2}$. Since $\left|E\left(C_{\mathscr{L}}\right) \cap E\left(D_{\mathscr{L}}\right)\right|=0$, each circle $D_{1}^{*}$ and $D_{2}^{*}$ and is contained in either $\mathrm{T}_{1}$ or $\mathrm{T}_{2}$. We separate the remainder of the proof into three subcases: in Case 3.1, $D_{1}^{*}$ and $D_{2}^{*}$ are both contained in $\mathrm{T}_{1}$; in Case 3.2, $D_{1}^{*}$ and $D_{2}^{*}$ are both contained in $\mathrm{T}_{2}$; and in Case 3.3, $D_{1}^{*}$ is contained in $\mathrm{T}_{1}$ and $D_{2}^{*}$ is contained in $\mathrm{T}_{2}$. In all three of these subcases, let $C_{\gamma}$ be a circle formed by connecting the endpoints of $\gamma$ in $\mathrm{T}_{2}$. Since $C_{\gamma}$ intersects each of $C_{1}$ and $C_{2}$ transversely at one point, $\vec{C}_{\gamma} \cdot \vec{C}_{1}= \pm \vec{C}_{\gamma} \cdot \vec{C}_{2}= \pm 1$; furthermore, since there can only be one relatively prime pair of integers, up to negation, satisfying this dot product relation, the homology class of $\vec{C}_{\gamma}$ is uniquely determined, up to negation.

Case 3.1: Here $\vec{C}_{\gamma} \cdot \vec{D}_{1}^{*}= \pm \vec{C}_{\gamma} \cdot \vec{D}_{2}^{*}= \pm 1$. Thus, since $D_{1}^{*}$ and $D_{2}^{*}$ are both contained in $\mathrm{T}_{1}, \sum_{j=1}^{2}\left|E(\gamma) \cap E\left(D_{j}\right)\right| \geqslant 2$. Thus $|E(C) \cap E(D)| \geqslant 2$.

Case 3.2: Since $\gamma$ is contained in $\mathrm{T}_{1}, \gamma \cap D_{\mathscr{L}}^{*}=\emptyset$. Thus $E(C) \cap E\left(D_{\mathscr{L}}=\emptyset\right.$. Now either $D=D_{\mathscr{L}}$ or $D$ has a connecting path of $\gamma_{D}^{*}$. If $D=D_{\mathscr{L}}$, then we are done; otherwise, since $\gamma_{D}^{*}$ has both endpoints in $T_{1},\left|\gamma_{D}^{*} \cap C\right| \neq 1$. Thus $|E(C) \cap E(D)| \neq 1$.

Case 3.3: In this case, $D_{1}^{*}$ and $D_{2}^{*}$ must be vertex-disjoint. Thus $D$ is a loose handcuff with connecting path $\gamma_{D}^{*}$ that has one endpoint in $\mathrm{T}_{1}$ and the other in $\mathrm{T}_{2}$. Thus $\left|\gamma_{D}^{*} \cap C_{\mathscr{L}}\right| \geqslant 1$. Just as for $C_{\mathscr{L}}, D_{\mathscr{L}}^{*}$ separates the torus into two connected components, $\mathrm{T}_{1}^{\prime}$ and $\mathrm{T}_{2}^{\prime}$, say. Since $D_{1}^{*}$ is contained in $\mathrm{T}_{1}$ and $D_{2}^{*}$ is contained in $\mathrm{T}_{2}$, one can show that $C_{1}$ is contained in $\mathrm{T}_{j}^{\prime}$ and $C_{2}$ is contained in $\mathrm{T}_{k}^{\prime}$, for $j \neq k$. Thus the connecting path $\gamma$ has one endpoint in $\mathrm{T}_{1}^{\prime}$ and the other in $\mathrm{T}_{2}^{\prime}$. Hence $\left|\gamma \cap D_{\mathscr{L}}^{*}\right| \geqslant 1$ and $|E(C) \cap E(D)| \geqslant\left|\gamma_{D}^{*} \cap C_{\mathscr{L}}\right|+\left|\gamma \cap D_{\mathscr{L}}^{*}\right| \geqslant 2$.

Theorem 4.2. If $G$ is 2-cell embedded in the torus, then $\mathscr{B}^{*}(\mathscr{A}(G))=\mathscr{B}\left(\mathscr{A}\left(G^{*}\right)\right)$. 
Proof. First, $\operatorname{rk}(\mathscr{B}(\mathscr{A}(G)))+\operatorname{rk}\left(\mathscr{B}\left(\mathscr{A}\left(G^{*}\right)\right)\right)=|E(G)|$ follows from the same argument as in the first paragraph of the proof of Theorem 3.1.

Second, if $C$ is a bias circuit of $\mathscr{A}(G)$ and $D^{*}$ is a bias circuit of $\mathscr{A}\left(G^{*}\right)$, then we can show that $|C \cap D| \neq 1$ by the same arguments as in the proof of Theorem 4.1, because a bias circuit in $\mathscr{A}(G)$ is a bias circuit in $\mathscr{H}(G)$ unless it is a balanced circle. In the case where $C$ is a balanced circle, $i(\vec{C}) \cdot(1,1)$ is even but $i(\vec{C}) \neq \mathbf{0}$. This implies that $\left|C \cap D_{\mathscr{L}}^{*}\right| \geqslant 2$ unless $D^{*}$ is a balanced circle in $\mathscr{A}(G)$. But when $D$ is a balanced circle, this implies that $C$ and $D^{*}$ are both lift circuits. Thus $\left|C \cap D^{*}\right| \neq 1$.

\section{Concluding remarks}

Similar matroid duality results should also hold for connected graphs that are 2-cell embedded in the Klein bottle. For duality results coming from graphs embedded in surfaces $\mathrm{S}$ of negative Euler characteristic, new matroids of rank $|V(G)|+\left\lceil\frac{2-\chi(\mathrm{S})}{2}\right\rceil$ need to be identified.

\section{References}

[1] Oxley, J. G. (1992) Matroid Theory, Oxford University Press.

[2] Stillwell, J. (1993) Classical Topology and Combinatorial Group Theory, 2nd edn, Springer.

[3] Zaslavsky, T. (1981) Characterizations of signed graphs. J. Graph Theory 5 401-406.

[4] Zaslavsky, T. (1989) Biased graphs I: Bias, balance, and gains. J. Combin. Theory Ser. B 47 $32-52$.

[5] Zaslavsky, T. (1991) Biased graphs II: The three matroids. J. Combin. Theory Ser. B 51 46-72.

[6] Zaslavsky, T. Personal communications. 